\title{
Development and optimization of erythromycin-loaded lipid-based gel by Taguchi design: In vitro characterization and antimicrobial evaluation
}

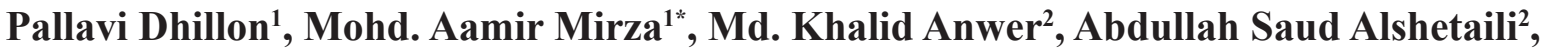 \\ Saad Maria Alshahrani ${ }^{\oplus 2}$, Zeenat Iqbal ${ }^{1 *}$

\begin{abstract}
${ }^{1}$ Department of Pharmaceutics, SPER, Jamia Hamdard University, New Delhi, India, ${ }^{2}$ Department of Pharmaceutics, College of Pharmacy, Prince Sattam Bin Abdulaziz University, Al-kharj, Saudi Arabia
\end{abstract}

\begin{abstract}
The foremost aim of the current research was to prolong and sustain the release of erythromycin (ERY) by preparing a solid lipid nanoparticles (SLNs)-based gel formulation for the safe and effective treatment of acne. ERY-loaded SLNs were developed, and various process variables were optimized with respect to particle size, zeta potential, and entrapment efficiency using the Taguchi model. The average particle size, PDI, zeta potential, drug entrapment efficiency, and drug loading of optimized SLN (F4) were found to be $176.2 \pm 1.82 \mathrm{~nm}, 0.275 \pm 0.011,-34.0 \pm 0.84,73.56 \%$, and $69.74 \%$ respectively. The optimized SLN (F4) was successfully incorporated into the carbopol-based hydrogel. The in vitro release of ERY from the SLN gel and plain gel were compared and found to be $90.94 \%$ and $87.94 \%$ respectively. In vitro study of ERY-loaded SLN gel showed sustained delivery of drug from formulation thus enhancing the antimicrobial activity after 30 hours when compared to ERY plain gel.
\end{abstract}

Keywords: Erythromycin. Solid lipid nanoparticles. Nanogel. Taguchi model. Characterization. Diffusion disc.

\section{INTRODUCTION}

Solid lipid nanoparticles (SLNs) offer an attractive means of drug delivery, particularly for poorly watersoluble drugs. They blend the advantages of polymeric nanoparticles (Nadkar, Lokhande, 2010), emulsions, and liposomes (Loxley, 2009; Mishra, Patel, Tiwari, 2010). SLNs consist of the drug entrapped in a biocompatible lipid core and surfactant in the outer shell, offering a good alternative to polymeric systems (Ekambaram, Sathali, Priyanka, 2012) in terms of lower toxicity (Muller, Mader, Gohla, 2000). Moreover, the production process can be modulated for desired drug release, protection of drug degradation, and avoidance of organic solvents. The aforementioned advantages make SLNs a promising carrier system for optimal drug delivery (Helgason et al., 2009). ERY is a safe and effective agent for the treatment of acne. Furthermore, dibenzoyl peroxide's lipophilic

\footnotetext{
*Correspondence: Z. Iqbal. Nano-formulation Research Lab, Department of Pharmaceutics, Faculty of Pharmacy, Jamia Hamdard University, New Delhi - 110062, India. E-mail: ziqbaljh@yahoo.co.in. Mohd. Aamir Mirza. E-mail: aamir.jhu07@gmail.com, Mobile: 91-9213378765
}

nature enhances transport through sebaceous glands, with maximum penetration through acne follicles. ERY can be bonded with the SLN surface to facilitate drug targeting of the skin strata and increase the efficiency of the acne remedy (Mehnert, Mader, 2001).

Topical ERY is used for the treatment of inflammatory acne vulgaris that occurs due to activity against propioni bacterium acne (Manjunath, Enkateswarlu, 2004). It is slightly soluble in water and freely soluble in methanol. ERY base and triamcinolone acetonide are examples of topical drugs with poor dermal localization due to lipophilicity. SLNs could be suitable carriers for these drugs with a potential impact on their dissolution.

The Taguchi model has been successfully used for the optimization of process variables. The design of the experiments aims to reduce the experimental runs required for optimization. The Taguchi model design is based on a special set of orthogonal arrays to standardize fractional factorial designs. This approach reduces the size of the factorial design. An orthogonal array implies that the design is well-adjusted such that the factor levels are weighed equally. Each factor can be 
evaluated independently of the other factors. This allows the assessment of the effect of one factor without the interference of the effects of other factors (Taguchi, 1987).

The purpose of this study to develop ERY-loaded SLNs-based gel with the potential to sustain and delay the release of the drug. The developed gel may be suitable for the treatment of acne.

\section{MATERIAL AND METHODS}

\section{Material}

Glyceryl monostearate, Polaxomer 188, stearic acid, and Comparitol were obtained from HiMedia Lab, Mumbai, India. Lecithin was obtained from Spectrum Chemicals and erythromycine from Yarrow Chemicals, Mumbai, India. All the other reagents and solvents used were of analytical reagent grade.

\section{Formulation and optimization of SLNs}

\section{Selection of method for preparation of SLNS}

Three different methods were used for the preparation of ERY-loaded SLNs.

\section{Microemulsion Technique}

The lipid is melted, and a mixture of water, surfactant, and co-surfactant(s) was heated at the same temperature as the lipid. It was then added under mild stirring (1000 rpm) to the melted lipid. A transparent, thermodynamically stable system was formed, since the compounds were mixed in the correct ratio. This microemulsion was then dispersed in a cold aqueous medium $\left(2-3{ }^{\circ} \mathrm{C}\right)$ under mild mechanical mixing ensuring that the small size of the particle is due to the precipitation and not mechanically induced by a stirring process (Surender, Deepika, 2016).

\section{Solvent Emulsification/Evaporation Technique}

The lipid was dissolved in a water-miscible organic solvent (methanol and chloroform, 1:1) and the drug was dispersed in the lipid solution. It was then emulsified in an aqueous phase containing the surfactant and the co-surfactant. Upon evaporation of the solvent in a Rota evaporator, a nanoparticle dispersion was formed by the precipitation of the lipid in the aqueous phase (Ahlin, Kristl, Kobar, 1998).

\section{Solvent Emulsification Diffusion Technique}

Different amounts of drugs and lipids were taken - each dissolved in a $2 \mathrm{~mL}$ mixture of methanol and chlorofonn (1:1) separately (as internal oil phase).
Powdered ERY (50 mg) was dispersed in the above solution and sonicated for 2 minutes. The resulting dispersion was poured into a solution containing $1.5 \%$ $(\mathrm{w} / \mathrm{v})$ aqueous surfactant solution (PluronicF-68) and homogenized for 30 minutes at $4000 \mathrm{rpm}$ to form an o/w emulsion. After homogenization, the emulsion was poured into ice-cold water up to a volume of $50 \mathrm{ml}$ and stirred for 3 hours to diffuse the organic solvent into external aqueous phase water. The dispersion was then centrifuged at $12000 \mathrm{rpm}$ for 15 minutes (Sartorius FI8 K) to separate the solid lipid material containing the drug. This was then re-dispersed in a 1.5\% aqueous surfactant (Pluronic F-68) solution and sonicated for 10 minutes (Surender, Deepika, 2016).

\section{Optimization of formulation component and process variables}

\section{Screening of lipids for SLNS}

A constant amount of drug $(50 \mathrm{mg})$ was weighed and dispersed into the lipid solution in different ratios ranging from 1:1 to 1:10 of lipids, and the rest of the parameters were kept constant. The surfactant concentration was found to be $1 \% \mathrm{w} / \mathrm{w}$. It was then homogenized for 30 minutes with a stirring time for 3 hours and sonicated for 5 minutes.

\section{Optimization of drug lipid loading ratio}

A constant amount of drug $(50 \mathrm{mg})$ was weighed and dispersed into lipid in different ratios ranging from $1: 1$ to $1: 5$ of lipids and the rest of the parameters were kept constant. The surfactant concentration was $1 \% \mathrm{w} / \mathrm{w}$. It was homogenized for 30 minutes with a stirring time for 3 hours and then sonicated for 5 minutes.

\section{Optimization of surfactant concentration}

On the basis of the reported literature, the concentration of surfactant was optimized for a drug lipid ratio of $1: 2$. The ratios used for the formulation were $0.5 \%$, $1 \%, 1.5 \%$, and $2 \%$. The surfactant was added at the time of emulsification, and the concentration of the surfactant was optimized regarding the particle size and aggregation after 24 hours.

\section{Optimization of stirring time}

During the process of stirring, organic solvents diffuse into the aqueous phase, leading to the synthesis of SLNs. The speed and the time of stirring may influence the particle size as well as the drug entrapment. In the present study, the stirring speed was kept constant at $3000 \mathrm{rpm}$ (calibrated by thread method), and the time of 
stirring was optimized. Three points of time were used for the optimization - 30, 45, and 60 minutes - at a constant surfactant concentration of $2 \%$, a drug: lipid ratio of $1: 2$, and a sonication time of 4 minutes.

\section{Optimization of probe sonication}

The probe sonicator (Bandelin Sonoplus, Biomate India) was used to optimize the sonication time viz 5,10 , 15 , and 20 minutes with the following parameters $5 \times 10$ cycle and $50 \%$ power.

\section{Design of experiments by the Taguchi model}

This involves the factorial design for optimization of process variables (Nazzal, Khan, 2002). On the basis of the literature reported, the aforementioned series of experiments were performed to identify the controlling factor and the noise factor. There are various factors that seem to affect the formulation. An experimental design is a statistical technique used to simultaneously analyze the influence of multiple factors on the properties of the system being studied. The purpose of an experimental design is to plan and conduct experiments in order to extract the maximum amount of information from the collected data in a minimal number of experimental runs. Factorial design, based on the response surface method, is applied to design formulations. However, an increase in the number of factors significantly increases the number of experiments that need to be carried out. The Taguchi approach proposes a special set of orthogonal arrays to standardize the fractional factorial design. This approach reduces the size of the factorial design, and a study can be performed with 9 sets of experiments for a three-level, four-factor $\left(3^{4}\right)$ design of experiments. The codes for all four variables at three different levels are illustrated in Table VII in the results and discussion section.

\section{Characterization of SLNs}

\section{Measurement particle size, zeta potential, and size distribution}

SLNs dispersions were diluted 50 times with the double distilled water for size determination and zeta potential measurement. Higher value of zeta potential may lead to disaggregation of particles in the absence of other complicating factors such as steric stabilizers or hydrophilic surface appendages. Zeta potential measurements allow predictions regarding the storage stability of colloidal dispersions (Yassin et al., 2010; Anwer et al., 2016a; Anwer et al., 2016b).

\section{In vitro drug release studies from SLNS}

In vitro release studies were performed in $\mathrm{pH} 6.4$ phosphate buffer using dialysis membrane (Mol. wt. 12000-14000 Dalton), and $5 \mathrm{~mL}$ of suspension was placed inside the dialysis tube, following which it was dipped the filled tube in buffer medium. The rpm of magnetic bead was 100 and the temperature was $37^{\circ} \mathrm{C}$. At time intervals $0,0.25$, $0.5,1,2,3,4,5,6,7,8,9,10,12$ and 24 hours, $1 \mathrm{~mL}$ aliquots were withdrawn, diluted suitably with fresh buffer solution, and analyzed for the drug content spectrophotometrically at $483.5 \mathrm{~nm}$ (Bhadra, Prajapati, Bhadra, 2016). The in vitro drug release was performed in triplicate. The concentration of ERY in test samples was calculated using a regression equation of the calibration curve.

\section{Development of optimized SLN-loaded gel}

Hydrogel base were prepared by carbopol 940. Carbopol resin were soaked in double distilled water $(10 \%$ Glycerin) for 12 hours and then dispersed by agitating at approximately $1000 \mathrm{rpm}$ with aid of mechanical stirrer for 10 minutes to get a smooth dispersion. Stirring was stopped and dispersion was allowed to stand so that any entrained air could escape. At this stage, optimized ERYloaded SLN was incorporated into gel with continuous stirring for 10 minutes. Any entrapped air in the gel was allowed to escape by allowing the gels to stand overnight (Bisht et al., 2017).

\section{Evaluation of hydrogel}

The developed gel was evaluated for their clarity, $\mathrm{pH}$, viscosity, spreadability, extrudability, occlusion effect, and in vitro drug release (Bisht et al., 2017).

\section{Clarity}

The clarity of developed gel formulation was determined by visual inspection under black and white background and it was graded as follows;

Turbid: +, clear: ++, very clear (glassy): +++ .

$\mathrm{pH}$

2.5 gm of gel was accurately weighed and dispersed in $25 \mathrm{ml}$ of distilled water. The $\mathrm{pH}$ of dispersion was measured by digital $\mathrm{pH}$ meter (Systronic $\mathrm{pH}$ system 362 ).

\section{Homogeneity}

All developed gels were tested for homogeneity by visual inspection after the gels have been set in the container for their appearance and presence of any aggregate. 


\section{Spreadability}

It was determined by wooden block and slide apparatus invented by Multimer et al.(1956). For the determination of spreadability, 1 gm of sample was applied in between two glass slide and was compressed to uniform thickness by placing some weight for 5 minutes. Weight $(50 \mathrm{gm})$ as added to pan. The time required to separate the two slides, i.e. the time in which the upper glass slide moves over the lower plates was taken as measure of spreadability (S).Time (T) taken to separate the slide completely from each other Viscosity measurement. The value of spreadability indicates that the gel is easily spreadable by small amount of shear.

Spreadability was calculated by using the formula:

$$
\mathrm{S}=\mathrm{ML} / \mathrm{T}
$$

where $\mathrm{S}=$ Spreadability; $\mathrm{M}=$ Weight tide to upper slide; $\mathrm{L}=$ Length moved on the glass slide; $\mathrm{T}=$ Time.

\section{Extrudability}

The extrudability test was carried out by using Pfizer tester, $10 \mathrm{gm}$ of gel was filled in aluminum tube. The plunger was adjusted to hold the tube properly. The pressure of $1 \mathrm{Kg} / \mathrm{gm}^{2}$ was applied for $30 \mathrm{sec}$. The quantity of gel extruded was weighed. The procedure was repeated at three equidistance places of the tube. Test was carried out in triplicates.

\section{Viscosity}

Viscosity of the gels was determined using a Brookfield viscometer, by using small sample adapter having spindle number SC4/13R (Middleboro, MA, USA). The gel was subjected to torque ranging from 10 to $100 \%$. The viscosity of various formulation ERY hydrogel was measured using a Brookfield viscometer.

\section{In vitro drug release from SLN gel}

In vitro release studies were performed in phosphate buffer ( $\mathrm{pH}$ 6.4) using dialysis membrane (Mol wt. 12000-14000 Dalton), and 0.5 gm of ERY-loaded plain gel as well as ERY SLN-loaded gel were placed inside the dialysis bag and dipped in a tube containing buffer medium. The tubes were placed in the biological shaker after setting $100 \mathrm{rpm}$ and temperature $37^{\circ} \mathrm{C}$. Aliquots $(1 \mathrm{~mL})$ were withdrawn at time intervals $(0.5,1,2,3,4,5$, $6,7,8,10,12$, and 24) and replaced by an equal volume of fresh dissolution medium. The samples were analyzed spectrophotometrically in triplicate at $483.5 \mathrm{~nm}$ after suitable dilution (Bhadra, Prajapati, Bhadra, 2016). The in vitro release data was fitted according to different kinetic models to analyze the release behavior from SLN-loaded gel (Bisht et al., 2017).

\section{In vitro antimicrobial activity: Disk diffusion method}

Disk diffusion refers to the diffusion of an antimicrobial agent of a specified concentration from disks, tablets, or strips into a solid culture medium that has been seeded with the selected inoculum isolated in a pure culture. Disk diffusion is based on the determination of an inhibition zone that is proportional to the bacterial susceptibility and the antimicrobial present in the disk. The diffusion of the antimicrobial agent into the seeded culture media results in a gradient of the antimicrobial agent. When the concentration of the antimicrobial agent becomes so diluted it can no longer inhibit the growth of the test bacterium, the zone of inhibition is demarcated. The diameter of this zone of inhibition around the antimicrobial disk is related to minimum inhibitory concentration (MIC) for that particular bacterium/antimicrobial combination. The zone of inhibition correlates inversely with the MIC of the test bacterium. Generally, the larger the zone of inhibition, the lower the concentration of antimicrobial agent required to inhibit the growth of the organisms. However, this depends on the concentration of antibiotic in the disk as well as its diffusibility.

The in vitro antibacterial activities of plain gel and SLN-loaded gel were performed against $\mathrm{S}$. aureus by disc diffusion method. Under aseptic conditions, empty sterile discs were impregnated with $50 \mathrm{mg}$ of ERY SLN-loaded gel as well as ERY plain gel and placed on the surface of the agar using sterile forceps. All petri dishes containing the microorganisms were sealed and incubated for 48 hours in a temperature of $37^{\circ} \mathrm{C}$. After the incubation period, the diameter of the inhibition zones was observed where clear zones were seen on the agar and measured using a ruler to the nearest millimeter readings. The test was performed in triplicates, and mean values of the diameters of the inhibition zones were calculated for both samples (Balouiri, Sadiki, Ibnsouda, 2016).

\section{RESULTS AND DISCUSSION}

\section{Formulation and optimization of SLN}

\section{Selection of preparation of method}

The microemulsion technique was selected as the method of choice in the formulation of solid lipid nanoparticles, because it showed better results in terms 
of particle size, particle shape, drug entrapment etc. as compared to the other methods, as seen in Table I. The versatility and flexibility of this method allows for the use of different lipids and drug candidates. In the present study, the lipid glyceryl monostearate was used for the preparation of ERY-loaded SLNs.

TABLE I - Comparison between different methods used for the preparation of SLNs

\begin{tabular}{|c|c|c|}
\hline Methods & Lipids & Particle Size (nm) \\
\hline \multirow{3}{*}{$\begin{array}{l}\text { Solvent } \\
\text { Evaporation } \\
\text { Method }\end{array}$} & Stearic acid & 1428 \\
\hline & GMS & 998 \\
\hline & $\begin{array}{c}\text { Comparitol ATO } \\
888\end{array}$ & 1272 \\
\hline \multirow{3}{*}{$\begin{array}{l}\text { Microemulsion } \\
\text { Technique }\end{array}$} & Stearic acid & 391 \\
\hline & GMS & 176.2 \\
\hline & $\begin{array}{c}\text { Comparitol ATO } \\
888 \\
\end{array}$ & 380.8 \\
\hline Solvent & Stearic acid & 642 \\
\hline Emulsification & GMS & 519 \\
\hline $\begin{array}{l}\text { Diffusion } \\
\text { Technique }\end{array}$ & $\begin{array}{c}\text { Comparitol ATO } \\
888\end{array}$ & 528 \\
\hline
\end{tabular}

\section{Optimization of formulation component}

\section{Screening of lipids for SLNS}

Three lipids - stearic acid, glycerol monostreate, and Comparitol 888 - were used for the formation of SLNs. A drastic increase in the particle size and decrease in drug entrapment was observed with the use of Comparitol 888 and stearic acid. Among these lipids, glyceryl monostearate was selected for the development of SLNs as mentioned in Table II. GMS had the particle size within the nanoparticle range and the demonstrated maximum drug entrapment.

TABLE II - Screening of lipids for SLNs

\begin{tabular}{lccc}
\hline Lipids & $\begin{array}{c}\text { Particle Size } \\
(\mathbf{n m})\end{array}$ & $\begin{array}{c}\text { Poly } \\
\text { dispersity } \\
\text { Index (PDI) }\end{array}$ & $\begin{array}{c}\text { Entrapment } \\
\mathbf{( \% )}\end{array}$ \\
\hline Stearic Acid & $1391 \pm 4.76$ & $0.569 \pm 0.06$ & $48.24 \pm 1.39$ \\
GMS & $176.2 \pm 0.48$ & $0.275 \pm 0.05$ & $73.56 \pm 0.28$ \\
Comparitol & $380.8 \pm 1.67$ & $0.412 \pm 0.02$ & $57.29 \pm 0.81$ \\
888 ATO & & & \\
\hline
\end{tabular}

Optimization of drug lipid loading ratio

A drastic increase in the particle size and decrease in the drug entrapment was observed when the drug: lipid ratio was increased from 1:1 to $1: 5 \mathrm{w} / \mathrm{w}$. The results indicate that the optimal drug lipid ratio is $1: 2 \mathrm{w} / \mathrm{w}$, as depicted in Table III. The increase in particle size and decrease in drug entrapment was probably caused by the increase in lipid quantity, which resulted in drug expulsion due to the crystalline structure of lipid.

TABLE III - Optimization of drug lipid ratio

\begin{tabular}{ccc}
\hline Drug Lipid Ratio & $\begin{array}{c}\text { Particle Size } \\
\text { (in nm) }\end{array}$ & $\begin{array}{c}\text { Drug Entrapment } \\
\text { (\%) }\end{array}$ \\
\hline $1: 1$ & $159 \pm 0.91$ & $44.2 \pm 0.36$ \\
$1: 2$ & $176 \pm 0.86$ & $78.1 \pm 0.74$ \\
$1: 3$ & $476 \pm 1.75$ & $55.3 \pm 1.03$ \\
$1: 4$ & $508 \pm 2.56$ & $22.4 \pm 0.55$ \\
$1: 5$ & $719 \pm 3.21$ & $34.5 \pm 0.87$ \\
\hline
\end{tabular}

\section{Optimization of surfactant concentration}

The concentration of the surfactant was optimized in order to obtain the smallest possible SLNs with maximum percentage of drug entrapment. The optimized one had $0.5 \%$ concentration of surfactant, as it lead to the smallest particle size, zeta potential within range, and it did not cause aggregation after 24 hours, as depicted in Table IV.

TABLE IV - Optimization of Surfactant Concentration

\begin{tabular}{cccc}
\hline $\begin{array}{c}\text { Surfactant } \\
\text { Conc. }\end{array}$ & $\begin{array}{c}\text { Particle Size } \\
(\mathbf{n m})\end{array}$ & $\begin{array}{c}\text { Zeta Potential } \\
(\mathbf{m V})\end{array}$ & $\begin{array}{c}\text { Aggregation } \\
\text { (After 24 h) }\end{array}$ \\
\hline $0.5 \%$ & $176 \pm 1.87$ & $24.42 \pm 0.95$ & - \\
$1.0 \%$ & $213 \pm 1.78$ & $25.26 \pm 1.02$ & - \\
$1.5 \%$ & $224 \pm 2.12$ & $24.12 \pm 0.59$ & + \\
$2.0 \%$ & $278 \pm 1.27$ & $24.38 \pm 1.01$ & + \\
\hline
\end{tabular}

\section{Optimization of stirring time}

During stirring, the organic solvent diffused into the aqueous phase, leading to the synthesis of SLNs. The speed and time of stirring may influence the particle size as well as the drug entrapment. Upon increasing the stirring time from 30 to 60 minutes, a decrease in particle size from 528 to $176 \mathrm{~nm}$ and an increase in entrapment i.e. $78.59 \%$ was observed (Table V).

\section{Optimization of Sonication time}

The sonication time was optimized by using the Bandlin Sonoplus by Biomate India, and 20 minutes of sonication was found to be the optimum time to reduce the particle size (Table VI). 
TABLE V - Optimization of Stirring Time

\begin{tabular}{ccc}
\hline Time (min.) & Particle Size (nm) & $\begin{array}{c}\text { Drug Entrapment } \\
\text { (\%) }\end{array}$ \\
\hline 30 & $528 \pm 3.29$ & $59.94 \pm 0.50$ \\
45 & $213 \pm 2.25$ & $64.73 \pm 0.18$ \\
60 & $176 \pm 1.73$ & $78.59 \pm 0.12$ \\
\hline
\end{tabular}

TABLE VI - Showing Optimization of Probe Sonication

\begin{tabular}{ccc}
\hline $\begin{array}{c}\text { Sonication time } \\
(\mathrm{min})\end{array}$ & Particle size $(\mathrm{nm})$ & Drug Loading \\
\hline $\mathbf{5}$ & $217.6 \pm 1.78$ & $59.94 \pm 0.54$ \\
$\mathbf{1 0}$ & $204.5 \pm 2.16$ & $63.23 \pm 0.76$ \\
$\mathbf{1 5}$ & $189.8 \pm 1.09$ & $68.92 \pm 0.52$ \\
$\mathbf{2 0}$ & $188.9 \pm 1.42$ & $72.79 \pm 0.12$ \\
\hline
\end{tabular}

\section{Design of experiment by Taguchi model}

The Taguchi model was used for simultaneous optimization of all variables used in the design of various nine formulations (F1-F9). All these variables were used at three different levels. The results in terms of particle size, polydispersity index, zeta potential, and drug entrapment efficiency are tabulated in Table VIII. In Formulation F1 lump formation occurred after 24 hours, therefore, the batch was discarded. F4 led to the smallest particle size with the highest drug loading capacity, therefore this batch was used as the optimized formulation. The codes for all four variables at three different levels were shown in the Table VII. On the basis of particle size, batch 4 is taken as optimized, hence the optimized parameter was found to be A2B1C2D3 as shown in above mentioned Table VII.

\section{Measurement particle size, zeta potential, and size distribution}

The particle size of formulation was found in the range of 176.2 to $374 \mathrm{~nm}$, as shown in Table VIII. Formulation factors like lipid amount and poloxamer
188 concentration were found to influence the particle size of the formulation significantly. Zeta potential (ZP) is the charge on a particle surface, and it is the inherent property of a particle. ZP plays a major role in the stability of multi-particulate liquid systems. It does not allow the particles to come in contact with each other and prevents aggregation thereby stabilizing the system. The ZP of glycerin monostreate-based SLN was found to be -34.0 indicating a stable formulation. PDI measures the particles size distribution in a system. This indicates the variation/ dispersion in particle size in SLN dispersion. The PDI values of the developed SLNs were found to be less than 1, confirming monodisperse particles (Table VIII).

\section{In vitro drug release studies from SLNs}

As seen in Figure 2, F4 was selected as the optimized formulation. It was clear from all the formulations that there was an initial burst release ranging from $5.879 \%(\mathrm{~F} 2)$ to $15.773 \%$ (F7). This was due to surface-absorbed drug on SLNs, which was followed by a sustained release, varying from $73.18 \%(\mathrm{~F} 8)$ to $92.90 \%$ (F4) as shown in Figure 2. This is due to the drug slowly diffusing through the lipid core. Thus, the formulation $\mathrm{F} 4$ was optimized as it released $93 \%$ of the drug in 24 hours. It was revealed from the results that ERY-loaded SLNs showed a slow release at pH 6.4 with a sustained pattern of release.

\section{Evaluation of ERY SLN-loaded gel formulation}

ERY SLN gel prepared with Carbopol 940 was evaluated for crucial parameters as listed in Table VIII. The gel demonstrated the desired homogeneity and viscosity as well as high spreadability and extrudability for the formulation. The rheological behavior of gel systems was studied. In a gel system, the consistency depends on the ratio of solid fraction, which produces the structure of the liquid fraction. The viscosity of ERY-loaded SLNs gel was found to be $9563 \pm 7.48$ centipoises. The gel was found to be uniform with pourable viscosity.

TABLE VII - Showing codes for all variables at three different level

\begin{tabular}{lccc}
\hline \multirow{2}{*}{ Variables } & \multicolumn{3}{c}{ Levels } \\
\cline { 2 - 4 } & Low & Medium & High \\
\hline Drug to lipid ratio & A1 $(1: 1)$ & A2 $(1: 2)$ & A3 (1:3) \\
Conc. of Surfactants & B1 $(0.5 \%)$ & B2 $(1 \%)$ & B3 $(1.5 \%)$ \\
Stirring time (min.) & C1 $(30 \mathrm{~min})$ & C2 $(45 \mathrm{~min})$ & C3 $(60 \mathrm{~min})$ \\
Sonication time (min.) & D1 $(10 \mathrm{~min})$ & D2 $(15 \mathrm{~min})$ & D3 $(20 \mathrm{~min})$ \\
\hline
\end{tabular}


TABLE VIII - Codes for four variables at three different levels

\begin{tabular}{|c|c|c|c|c|c|c|c|c|c|}
\hline \multirow{2}{*}{ Codes } & \multicolumn{4}{|c|}{ Parameters } & \multirow{2}{*}{$\begin{array}{l}\text { Particle Size } \\
\quad(\mathrm{nm})\end{array}$} & \multirow{2}{*}{ PDI } & \multirow{2}{*}{$\mathbf{Z P}(\mathbf{m V})$} & \multirow{2}{*}{ DL (\%) } & \multirow{2}{*}{ DE (\%) } \\
\hline & $\mathbf{A}$ & $\mathbf{B}$ & $\mathrm{C}$ & D & & & & & \\
\hline $\mathrm{F} 1$ & 1 & 1 & 1 & 1 & \multicolumn{5}{|c|}{ Lump Formation } \\
\hline $\mathrm{F} 2$ & 1 & 2 & 2 & 2 & $256.6 \pm 2.41$ & $0.314 \pm 0.008$ & $24.26 \pm 0.83$ & 34.29 & 31.29 \\
\hline F3 & 1 & 3 & 3 & 3 & $274.7 \pm 3.27$ & $0.374 \pm 0.012$ & $20.71 \pm 1.04$ & 60.19 & 68.42 \\
\hline $\mathrm{F} 4$ & 2 & 1 & 2 & 3 & $176.2 \pm 1.82$ & $0.275 \pm 0.011$ & $-34.0 \pm 0.84$ & 69.73 & 73.56 \\
\hline F5 & 2 & 2 & 3 & 1 & $209.4 \pm 2.48$ & $0.496 \pm 0.032$ & $20.14 \pm 0.86$ & 59.37 & 71.97 \\
\hline F6 & 2 & 3 & 1 & 2 & $215.8 \pm 3.23$ & $0.472 \pm 0.061$ & $-23.94 \pm 1.03$ & 61.19 & 71.38 \\
\hline F7 & 3 & 1 & 3 & 2 & $374.3 \pm 4.17$ & $0.561 \pm 0.005$ & $-23.21 \pm 1.05$ & 58.21 & 52.73 \\
\hline F8 & 3 & 2 & 1 & 3 & $327.9 \pm 3.20$ & $0.673 \pm 0.083$ & $-25.25 \pm 0.95$ & 54.80 & 36.69 \\
\hline F9 & 3 & 3 & 2 & 1 & $319.2 \pm 4.15$ & $0.712 \pm 0.023$ & $-24.69 \pm 1.10$ & 54.96 & 38.14 \\
\hline
\end{tabular}

TABLE IX - Evaluation of ERY loaded SLN gel

\begin{tabular}{lccccc}
\hline Clarity & $\begin{array}{c}\text { Mean } \mathbf{p H} \pm \text { S.D. } \\
(\mathbf{n}=\mathbf{3})\end{array}$ & Homogenity & $\begin{array}{c}\text { Mean Viscosity } \\
(\text { Pa.s }) \pm \text { S.D. }(\mathbf{n}=\mathbf{3})\end{array}$ & $\begin{array}{c}\text { Mean spreadability } \\
(\mathbf{g c m} / \mathbf{s e c}) \pm \text { S.D. }(\mathbf{n}=\mathbf{3})\end{array}$ & $\begin{array}{c}\text { Mean Extrudability } \\
(\mathbf{g m}) \pm \text { S.D. }(\mathbf{n}=\mathbf{3})\end{array}$ \\
\hline++ & $6.5 \pm 0.34$ & Good & $9563 \pm 7.48$ & $19.51 \pm 0.71$ & $231 \pm 4.65$ \\
\hline
\end{tabular}

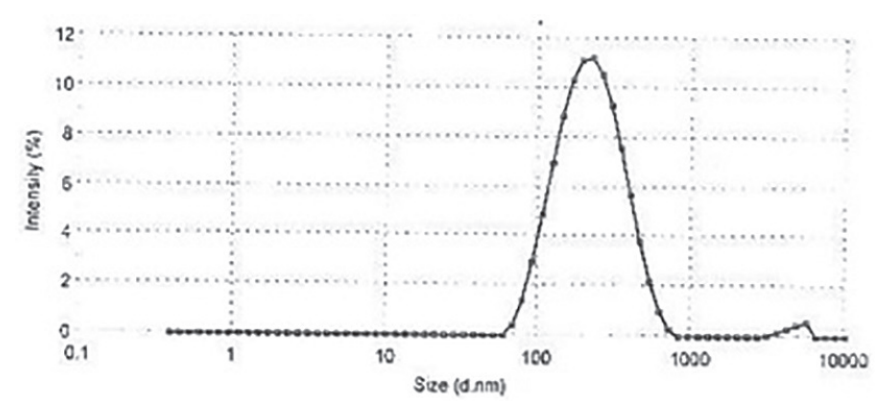

FIGURE 1 - Particle size of optimized formulation (F4).

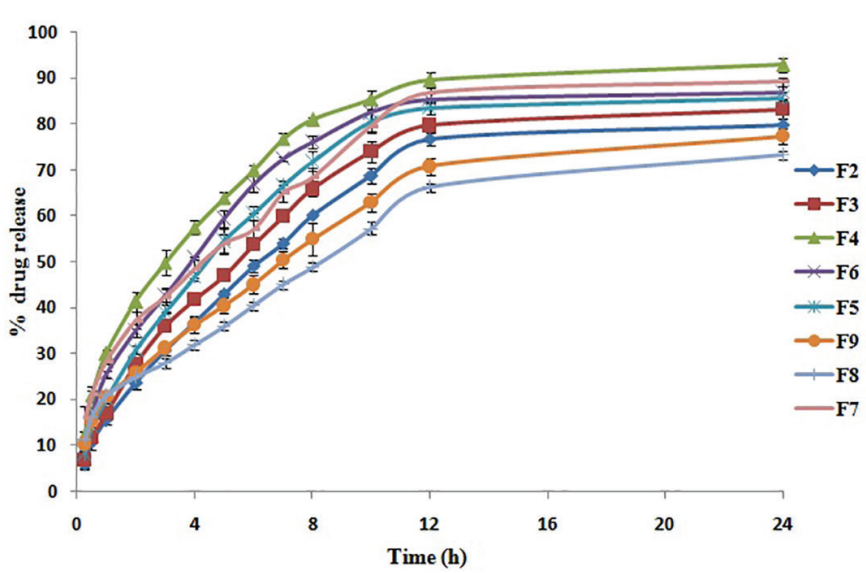

FIGURE 2 - In vitro release profile of developed SLNs.

\section{In vitro release profile of gel}

The in vitro release profile (Figure 3) of ERY plain gel $(2 \% \mathrm{w} / \mathrm{w})$ and ERY SLN-loaded gel $(2 \% \mathrm{w} / \mathrm{w})$ are seen in Figure 3. The percentage of cumulative drug release of plain gel and ERY-loaded SLN gel were found to be $90.94 \%$ and $87.94 \%$ respectively after 24 hours. The release of ERY from SLN-loaded gel was found to be low as compared to plain gel. SLN gel showed slower and more sustained release as compared to ERY plain gel.

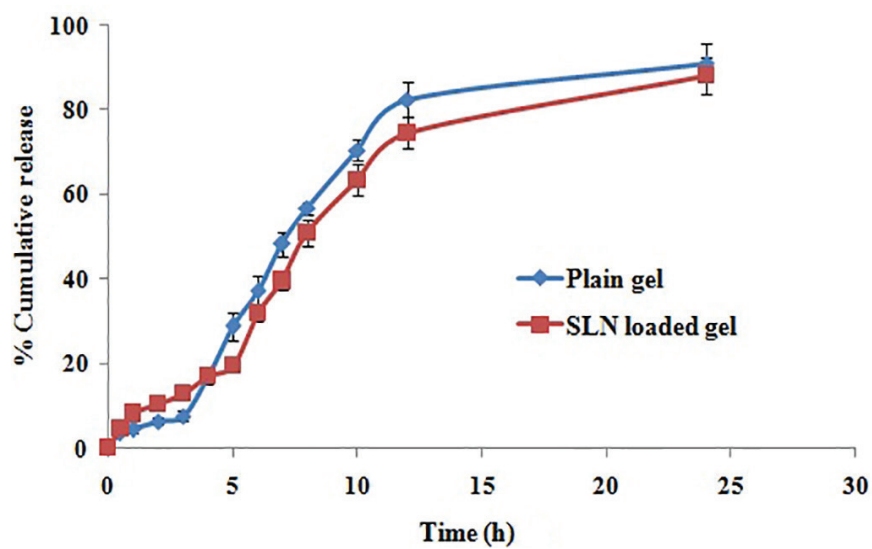

FIGURE 3 - In vitro release profile of developed Plain and SLN loaded gel.

The rate of the release of ERY SLN-loaded gel was studied by using various models. In the case of lipophilic matrices, swelling and erosion of polymers occurs simultaneously, and both contribute to the overall drug release rate. It was documented earlier that the drug release from lipophilic matrices shows a typical time-dependent 
profile (i.e. decrease of drug release with time due to increased diffusion path). This inherent limitation leads to first order release kinetic.

In our study, the formulation was designed for the controlled release of ERY, which was evaluated by in vitro drug release. To study the release kinetic of the drug, the result of the in vitro drug release studies were plotted with various kinetic models like zero order, first order, Kosermeyer and Peppas equation, and Higuchi's kinetics model. The regression values for the models used for ERYSLN formulations are mentioned in Table X.

TABLE X - Drug release kinetics

\begin{tabular}{cccc}
\hline Zero order & First order & Higuchi & $\begin{array}{c}\text { Korsemeyer- } \\
\text { Peppas }\end{array}$ \\
\hline $\mathbf{R}^{2}$ & $\mathbf{R}^{2}$ & $\mathbf{R}^{2}$ & $\mathbf{R}^{2}$ \\
\hline 0.955 & 0.966 & 0.981 & 0.782 \\
\hline
\end{tabular}

The result of the in vitro release study of SLN gel followed Higuchi kinetics $\left(\mathrm{R}^{2}=0.981\right)$. This correlates with the mechanism of drug release from a transdermal system. The Higuichi model was developed to depict the release of low soluble drugs when incorporated in semisolid and solid matrices (Bisht et al., 2017).

\section{In vitro antimicrobial activity}

A comparative evaluation of zone of inhibition of the prepared ERY SLN-loaded gel and ERY plain gel is mentioned in Figure 4. The zone of inhibiton of SLN-based gel was significantly higher as compared to plain gel. ERY-loaded SLNs gel can act as an effective therapeutic modality for treating acne by a decrease dose and frequency as well as improved patient compliance.

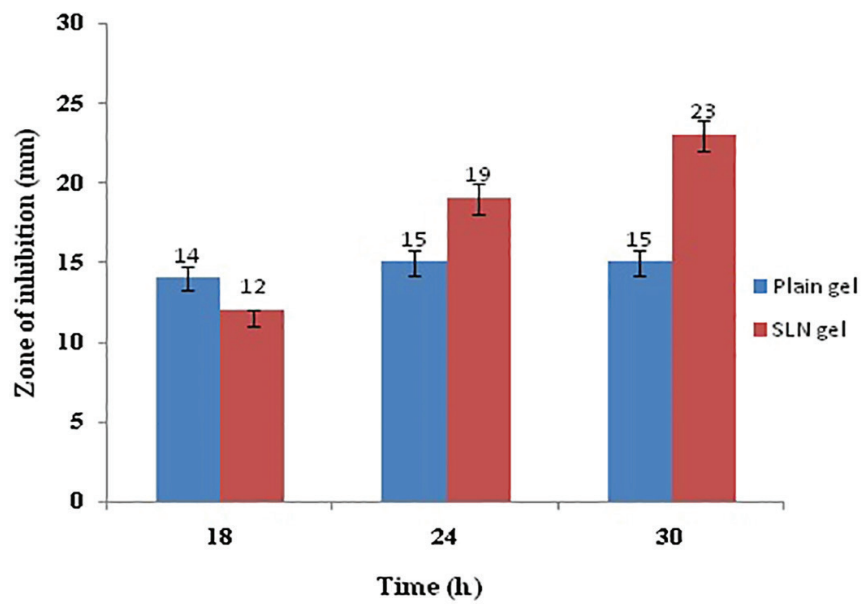

FIGURE 4 - Zone of inhibition by developed Plain and SLN loaded gel.

\section{CONCLUSION}

The ERY-loaded SLN gel was successfully incorporated into carbopol gel for topical delivery with a sustained release of drug. Carbopol $940(2 \% \mathrm{w} / \mathrm{v})$ gel was used as a hydrogel base with good spreadability and extrudability with compatible $\mathrm{pH}$. The in vitro release profile of erythromycin-loaded SLN gel showed a sustained pattern of drug delivery, and thus it enhances antimicrobial activity after 30 hours when compared with ERY plain gel. The obtained results suggest that the developed formulation benefits from its nano size and promises better therapeutic efficacy. ERY SNL-loaded gel can therefore be a good replacement for the conventional formulation with benefits of decreased dose and dosing frequency as well as improved patient compliance.

\section{ACKNOWLEDGEMENT}

The authors are thankful to Department of Pharmaceutics, Jamia Hamdard University, New Delhi, India for providing the essential facilities needed in this research.

\section{CONFLICT OF INTEREST STATEMENT}

The authors report no conflict of interest in this work. The author is responsible for content and the writing of the paper.

\section{REFERENCES}

Ahlin P, Kristl J, Kobar S. Optimization of procedure parameters and physical stability of solid lipid nanoparticles in the dispersion. Acta Pharm. 1998,48:257-67.

Anwer MK, Al-Mansoor MA, Jamil S, Al-Shdefat R, Ansari MN, Shakeel F. Development and evaluation of PLGA polymer-based nanoparticles of quercetin. Int J Biol Macromol. 2016a;92:213-9.

Anwer MK. Jamil S, Ansari MJ, Iqbal M, Imam F, Shakeel F. Development and evaluation of Olmesartan medoxomil-loaded PLGA nanoparticles. Mat Res Innovat. 2016b;20(3):193-97.

Balouiri M, Sadiki M, Ibnsouda SK. Methods for in vitro evaluating antimicrobial activity: A review. J Pharm Anal. 2016;6(2):71-9. 
Bhadra S, Prajapati AB, Bhadra D. Development of $\mathrm{pH}$ sensitive polymeric nanoparticles of erythromycin stearate. J Pharm Bio Allied Sci. 2016;8(2):135-40.

Bisht D, Verma D, Mirza MA, Anwer MK, Iqbal Z. Development of ethosomal gel of ranolazine for improved topical delivery: In vitro and ex vivo evaluation. J Mol Liquids. 2017;225:475-81.

Ekambaram P, Sathali AH, Priyanka K. Solid lipid nanoparticles: A review. Scient Rev Chem Comm. 2012;2(1):80-102.

Helgason T, Awad TS, Kristbergsson K, Me Clements DJ, Weiss J. Effect of surfactant surface coverage on formation of solid lipid nanoparticles (SLN). J Colloid Interface Sci. 2009;334(1):75-81.

Loxley A. Solid lipid nanoparticles for the delivery of pharmaceutical actives. Drug Deliv Technol. 2009;9(8):32.

Manjunath K, Venkateswarlu V. Preparation, characterization, and in vitro release kinetics of clozapine solid lipid nanoparticles. J Control Release. 2004;95(3):627-38.

Mehnert W, Mader K. Solid lipid nanoparticles: Production, characterization, and applications. Adv Drug Deliv Rev. 2001;47(2-3):165-96.

Mishra B, Patel BB, Tiwari S. Colloidal nanocarriers: A review on formulation technology, types, and applications toward targeted drug delivery. Nanomed Nano technol Biol Med. 2010;6(1):e9-e24.
Muller RH, Mader K, Gohla S. Solid lipid nanoparticles (SLN) for controlled drug delivery: A review of the state of the art. Eur J Pharm Bio pharm. 2000;50:161-77.

Mutimer MN, Riffkin C, Hill JA, Glickman ME, Cyr GN. Modern ointment bases technology II: Comparative evaluation of bases. J Am Pharm Assoc. 1956;45(4):212-18.

Nazzal S, Khan M. Response surface methodology for the optimization of ubiquinone self-nanoemulsifying drug delivery system. AAPS Pharm Sci Tech. 2002;3(1):23-31.

Nadkar S, Lokhande C. Current trends in novel drug delivery: An OTC perspective. Pharma Time. 2010;42(4):17-23.

Surender V, Deepika M. Solid lipid nanoparticles: A comprehensive review. J Chem Pharm Res. 2016;8(8):102-14.

Taguchi G. System of Experimental Design. New York: UNIPUB, Kraus International Publications; 1987.

Yassin AB, Anwer MK, Mowafy HA, El-Bagory IM, Bayomi MA, Alsarra IA. Optimization of 5-flurouracil solid-lipid nanoparticles: A preliminary study to treat colon cancer. Int $\mathrm{J}$ Med Sci. 2010;7(6):398-408.

Received for publication on $03^{\text {th }}$ July 2017 Accepted for publication on $21^{\text {st }}$ June 2018 